\title{
Development and Validation of the Biofrequency Applied Technique MMT (Molecular Music Therapy), with Consequent Application on A Cohort of Patients with A Wide Spectrum of Clinical Pictures
}

Stefano Turini ${ }^{1 *}$, Momir Dunjic ${ }^{2}$, Dejan Krstic ${ }^{3}$, Katarina Dunjic ${ }^{4}$, Marija Dunjic ${ }^{5}$, Petar Miodragovic ${ }^{6}$ and Milos Mirkovic $^{2}$

${ }^{1}$ School of Physiotherapy, AMEU-ECM University, Slovenia

${ }^{2}$ School of Medicine, University of Pristina in Kosovska Mitrovica, Serbia

${ }^{3}$ Faculty of Environmental Safety, University of Nis, Serbia

${ }^{4}$ School of Medicine, University of Belgrade, Serbia

${ }^{5}$ MMA Medical Faculty, University of Defence, Belgrade

${ }^{6}$ BDORT Center of Functional Supplementation and Integrative Medicine, Serbia

*Corresponding author: Stefano Turini, School of Physiotherapy, AMEU-ECM University, Italy

Keywords: Bio resonance frequency; MMT; Binaural tones; Sirtuin-1; Telomeres; TP-53; BDORT

\section{Introduction}

Everything that surrounds us vibrates and consequently emits a form of frequency that is not audible to the naked ear, since it falls within the range of ELF (Extreme Low Frequencies) waves, but it is possible to record it with appropriate sensitive instruments [1]. We need to imagine cells, atoms, molecules and subatomic particles, as entities that are not static but in constant motion; the single cells, as unicellular entities or forming a part of a tissue, in their movements, cause the emission of waves in the extracellular medium. These waves propagate in space, rich in water and solutes [2], meeting other cells. the same is true for the molecules that make up the cellular entities and for the same atoms that constitute these molecules. If it is possible to record the frequency of Nuclear Magnetic Resonance (NMR) of atomic nuclei, why would it not be possible to perform the same and record the bio resonance frequency for molecules and for the cells themselves? The question we asked ourselves in our research project is: can these extremely low frequencies have an effect on cellular functionality and on the structural-functional nature of the cells themselves? Studies conducted in past years have shown how ELF waves have an effect in regulating cell function [3].

A French scholar, physicist and musician, Joel Sternheimer, in the 1970s, wondered how music influenced plant growth and noticed, in his research project of recording bio resonance frequencies, with very sensitive instruments, of the single amino acids and nucleotides, that the frequencies of such molecules were similar to those of the musical notes, with the variation of few Hertz. He therefore created computerized matrices of association Amino Acid-Musical Note and Nucleotide-Musical Note [4]. In short, it was possible to convert the Primary Structure of a polypeptide or a protein or a nucleotide sequence of a specific segment of DNA and/or RNA, in a sequence of musical notes. After doing this, he converted the amino acid sequences of vegetable proteins useful for growth into music and sent them, like sounds, onto the vegetables themselves. The results led to the conclusion that plants treated with musical sequences derived from molecules grew more luxuriantly than untreated plants. 


\section{Material and Methods}

Encouraged by these results, we developed an artificial intelligence software, calling it SONGENPROT-SOLARIS, which implemented Joel Sternheimer's association matrices, with the variant, implemented directly within the software algorithm itself, that sound production occurred $30 \mathrm{~Hz}$ phase shift between the right headphone and the left headphone, of the system directly connected to the instrumentation, made by laptop Acer ASPIRE $7540 \mathrm{G}$ and Plantronics headphones. This allowed to create the phenomenon of Binaural Tones, capable of inducing a specific brain activity. We have therefore assembled a cohort of patients with a broad spectrum of clinical pictures (in total, we currently have 97 volunteer patients) in order to treat them with the sound emission of molecules such as: Sirtuin-1 (fundamental protein for regulation and scavenging of the radical oxygen and nitrogen species and the modulation of the oncosuppressor function and Telomeres length), Telomeres (repetitive TTAGGG sequences to the terminal portions of the chromosome arms, by not coding nature) and TP-53 (one of the most important tumor suppressors).

We therefore called this technique MMT or Molecular Music Therapy. The procedure consisted in placing the patient lying on a self-propelled bed, connected to an apparatus of headphones, directly connected with the laptop equipped with the specific software SONGENPROT-SOLARIS. The main purpose of our research project is to demonstrate the possibility of inducing genetic expression without the help of canonical stimulation with transcription factors, but only through the use of sound waves and electromagnetic frequencies. If a patient had low values of Sirtuin-1, Telomeres and/or, TP-53, determined by BDORT, then he/she would be treated with the sound sequence corresponding to these specific molecules, translated into music with the help of our software. The sound sequence, corresponding to the molecule in question, would stimulate the expression of the molecule gene itself, as a sort of genetic therapy based on the exclusive use of sounds.

\section{Results}

Before and after the treatment with the sound emission of the three molecules mentioned above, the patient was tested, using a BDORT [5,6], patented by Yoshiaki Omura in 1993, and applied, with plasticized functional slides, in order to perform a quantitative analysis on the three molecules described above (Sirtuin-1, Telomeres and TP-53). At the end of the MMT treatment we realized that the values of the three molecules increased (Figure 1) and this happened in subsequent treatments for the same patient (intrapatient determination) and in different patients, for the same pathology and for different clinical pictures (interpatient determination).
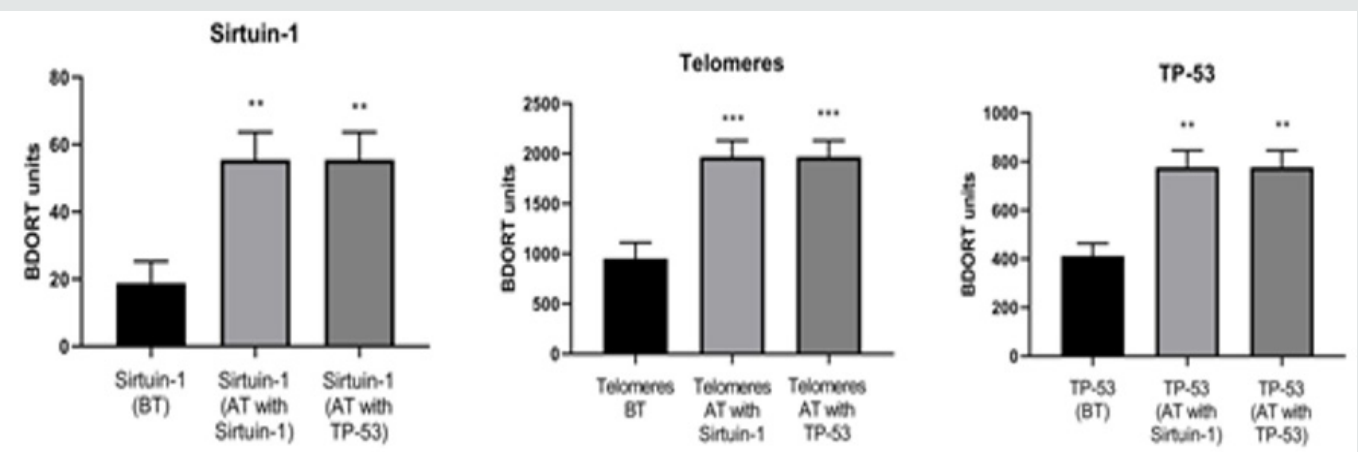

Figure 1: Summarized result of patients about three molecular parameters, detected Before Treatment (BT) and After Treatment (AT).

\section{Discussion}

The statistical re-elaboration of BDORT Test data, on the three molecular parameters determined before and after MMT treatment, was performed with Graph Pad Prism 8.0 statistical software. What we have obtained was a high degree of statistical correlation in the parameters measured after treatment (with $r<0,002^{* *}$ and $r<$ $0,001^{* * *}$ ). Through a series of treatments, the individual patients began to experience improvements at a psycho-physical level. Encouraged by these results, we decided to extend the research and perform epigenetic tests on patient blood samples, in order to confirm the results obtained with BDORT Test, on the three parameters taken into consideration (Sirtuin-1, Telomeres and TP53). Epigenetic research will serve the dual purpose of confirming the validity of the MMT technique and the BDORT kinesiological technique.

\section{Conclusion}

Following the results obtained, and statistical significance of parameters after treatment, compared with parameters before of treatment, determined by BDORT technique, we can confirm that MMT represents a valid tool for therapeutic application, for the treatment of a wide spectrum of pathologies.

\section{References}

1. Montagnier L, Aissa J, Del Giudice E, Lavallee C, Tedeschi A, et al. (2011) DNA waves and water. Journal of Physics Conference Series 306(1): 012007.

2. Foletti A, Ledda M, Lolli MG, Grimaldi S, Lisi A (2017) Electromagnetic information transfer through aqueous system. Electromagn Biol Med 36(3): 289-294.

3. Lisi A, Foletti A, Ledda M, Rosola E, Giuliani L, et al. (2006) Extremely Low Frequency $7 \mathrm{~Hz}, 100 \mathrm{~T}$ Electromagnetic Radiation Promotes 
Differentiation in the Human Epithelial Cell Line HaCaT. Electromagn Biol Med 25(4): 269-280.

4. Joël Sternheimer, "Procédé de régulation épigénétique de la biosynthèse des protéines par résonance d'échelle", brevet n ${ }^{\circ}$ FR 9206765 (1992), n ${ }^{\circ}$ de publication 2691796, aujourd'hui délivré en France (13/7/95) et 16 autres pays (dont OAPI, Australie, Russie).

5. Omura Y (2016) Clinical Significance of Human Papillomavirus Type 16 for Breast Cancer \& Adenocarcinomas of Various Internal Organs and
Alzheimer's Brain with Increased $\beta$-amyloid (1-42); Combined Use of Optimal Doses of Vitamin D3 and Taurine 3 times/day Has Significant Beneficial Effects of Anti-Cancer, Anti-Ischemic Heart, and Memory \& Other Brain Problems By Significant Urinary Excretion of Viruses, Bacteria, and Toxic Metals \& Substances. Acupunct Electrother Res 41(2):127-134.

6. Dunjic M, Stanisic S, Krstic D, Stanisic M, Jovanovic-Ignjatic Z, et al. (2014) Integrative Approach to Diagnosis of Genital Human Papillomaviruses (HPV) Infection of Female. Acupunct Electrother Res 39(3-4): 229-239.
(C) Commons Attribution 4.0 License

To Submit Your Article Click Here: Submit Article

DOI: 10.32474/OAJCAM.2019.01.000129

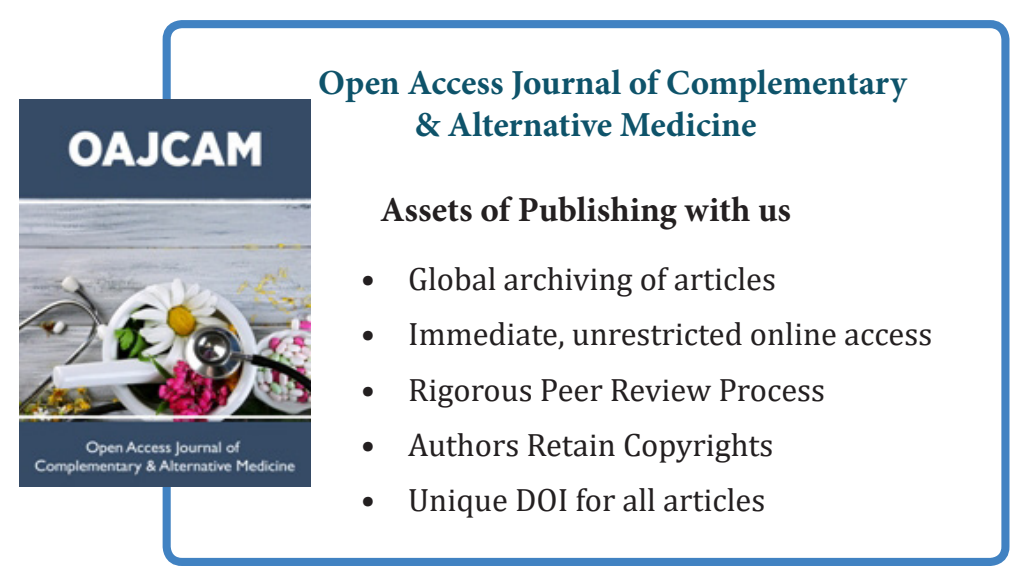

